\title{
Combinatory Analysis of Cell-free and Circulating Tumor Cell DNAs Provides More Variants for Cancer Treatment
}

\author{
SUN-YOUNG LEE ${ }^{1,2 \dagger}$, DONG-KYU CHAE ${ }^{3 \dagger}$, JAHYUN AN ${ }^{3 \dagger}$, SEOKCHAN YOO ${ }^{3}$, SUNGMOK JUNG $^{3}$, \\ CHANG HOON CHAE ${ }^{4}$, JONG BHAK ${ }^{3,5}$, BYUNG CHUL $\mathrm{KIM}^{3 *}$ and DONG-HYU CHO ${ }^{2,6^{*}}$ \\ Departments of ${ }^{1}$ Radiation Oncology and ${ }^{6}$ Obstetrics and Gynecology, \\ Chonbuk National University Hospital-Chonbuk National University Medical School, Jeonju, Republic of Korea; \\ ${ }^{2}$ Research Institute of Clinical Medicine of Chonbuk National University-Biomedical \\ Research Institute of Chonbuk National University Hospital, Jeonju, Republic of Korea; \\ ${ }^{3}$ Clinomics Inc., Suwon, Republic of Korea; \\ ${ }^{4}$ Department of Biophysics and Radiation Biology, Lab of Nanochemistry, Semmelweis University, Budapest, Hungary; \\ ${ }^{5}$ KOGIC, UNIST, Ulsan, Republic of Korea
}

\begin{abstract}
Background/Aim: Non-invasive biomarker detection using DNA from cell-free circulating DNA (cfDNA) and circulating tumor cells (ctcDNA) are emerging as they can be used for early diagnosis, prognosis and therapeutic target selection for cancer. However, cfDNA and ctcDNA from the same patient have not yet been compared extensively on how different the genetic characteristics of the two are in terms of the overlap between them. Materials and Methods: The performance of a customized NGS panel was used to compare the variants found in the 20 pairs of cfDNA and ctcDNA from gynecological cancer patients. Results: A genetic variant analysis revealed that there were only nine common overlapping variants out of 63 between the cfDNA and ctcDNA pairs, while 31 and 22 were unique to cfDNA and ctcDNA, respectively. Conclusion: A combinatory analysis of both cfDNA and CTCs from cancer patients can improve the sensitivity of liquid biopsies. These results are expected to provide better genetic target information for guiding clinical strategies for cancer. Tumor cells can acquire mutations even during cancer
\end{abstract}

These Authors contributed equally to this study.

*These Authors contributed equally to this study.

Correspondence to: Dong-Hyu Cho, MD, Department of Obstetrics and Gynecology, Chonbuk National University Medical School, 20, Geonjiro, Deokjin-gu, Jeonju, Jeonbuk 54907, Republic of Korea. Tel: +82 0632501360, Fax: +82 0632544833, e-mail: obgyn2001@jbnu.ac.kr; Byung Chul Kim, Ph.D., Clinomics Inc., Suite 201, Central Biz Tower, 260, Changyong-daero, Yeongtong-gu, Suwon-si, Gyeonggi-do 16229, Republic of Korea. Tel: +82 7082866966, Fax: +82 3180667274, e-mail: bckim@clinomics.co.kr

Key Words: NGS, cancer panel, liquid biopsy, cell free DNA (cfDNA), circulating tumor cells (CTC), gynecological cancer. therapies and can be selected for survival. Therefore, repeated monitoring of genetic mutations is important for tracking the condition of cancer patients. Genetic mutation profiles can be obtained by panel-based next generation sequencing (NGS) on tissue biopsy samples. Such profiles can provide ample information for choosing suitable drugs or chemotherapy. However, repeated solid tissue biopsies are invasive and inconvenient $(1,2)$. On the other hand, liquid biopsies including cell-free DNA (cfDNA), circulating tumor cells (CTCs) and tumor-derived exosomes from apoptotic or necrotic cells can be non-invasive and can also be sufficient enough to provide core information on the primary tumors or metastatic sites (3-5). For instance, one of the FDA approved commercial kits, Roche's Cobas plasma EGFR detection test V2 (US-IVD), utilizes cfDNA to detect a single mutation. In the case of next generation sequencing (NGS) application, Guardant360 now allows monitoring and aftercare of patients by screening cfDNA derived mutations for targeted therapies. As observed in many commercial products, liquid biopsy is an emerging field for real-time monitoring of tumor heterogeneity and predicting clinical behaviors of cancer in replace of tissue biopsy (6).

Currently, early detection of cancer using circulating tumor cells in patient's blood is limited by the small number of CTCs and the amount of blood drawn, and the sensitivity in isolating CTCs from normal tissue cells. By simply increasing the volume of blood, a higher amount of circulating tumor DNA (ctDNA) can be obtained. Guardant, for example, requires a total $40 \mathrm{ml}$ of blood to be drawn. Although this is an easy way to overcome the low amount issue, collecting a large blood volume is difficult in most clinical settings. Increasing the assay sensitivity is another approach to detect low amounts of ctDNA. Several methods, such as droplet digital PCR (ddPCR) and Beads, Emulsions, 
Amplification, Magnetics (BEAMing), have been devised to identify target tumor-specific mutations in cfDNA (7-9). However, the average cfDNA concentration in a healthy human subject is known to be only about $10 \mathrm{ng} / \mathrm{ml}$ and also the ratio of ctDNA to cfDNA is too diverse, representing from $0.01 \%$ to more than $50 \%$ of the cfDNA fraction (1013). The low fraction ratio of ctDNA indicates that the number of cancer-derived template DNA strands may not be sufficient even with $0.1 \%$ sensitivity. Therefore, simply increasing the sensitivity would not increase the detection rate of cancer-related mutations.

CTCs captured from blood are directly shed from the primary tumor or metastatic sites and can even be cultured for in-depth analyses providing much more definite information about cancer origin and status. Studies have reported advantages such as good concordance between genetic mutations in CTCs and primary tissues. These CTC mutations were found to provide more effective options to monitor tumor genomes that are prone to change during progression, treatment, and relapse $(14,15)$. As cfDNA and CTCs possess different analytical significances, the combinatory analysis of both samples from the same patient may provide complementary and additional information on tumor cell heterogeneity that is invaluable for identifying key molecular targets to improve cancer therapies. Recently, Onidani $\mathrm{K}$ et al. compared mutations detected in ctcDNA and cfDNA from patients with colorectal cancer or head and neck cancer. Their results demonstrated that ctcDNA expressed mutations in some blood samples were not detected in cfDNA or vice versa. In addition, their NGS data suggested that ctcDNA and cfDNA exhibit unique genetic alteration profiles (16). Therefore, we designed a different strategy to analyze not only the cfDNA mutations but also the genomic mutations of ctcDNA together in an effort to provide a richer set of data per patient. In this study, we analyzed the CTC genome present in the blood to compensate for the low cfDNA tumor fraction using ultra-deep next-generation sequencing utilizing a customized cancer panel that covers 51 actionable genes. As a result, we observed that the genetic variant profiles do not overlap significantly between cfDNA and ctcDNA. These results suggest that genetic analysis of either cfDNA or ctcDNA only misses certain genetic variants of tumors, and combined analysis on both types of DNA can possibly increase sensitivity and accuracy through ultra-deep sequencing.

\section{Materials and Methods}

Cell culture and DNA extraction. Human KOREF (Korean Reference genome) cell lines (17) and PC9 cells were cultured in RPMI- 1640 medium with $10 \%$ heat-inactivated fetal bovine serum. These prepared cells were incubated at $37^{\circ} \mathrm{C}$ and $5 \% \mathrm{CO}_{2}$. The DNA extracted from 100 cultured KOREF and PC9 cells was used as negative control and positive control, respectively. After ten or hundred PC9 cells were spiked into the normal blood, CTCs were
Table I. The 51 target genes in the NGS panel.

\begin{tabular}{lllllll}
\hline$A B L 1$ & BTK & ERBB3 & GNA11 & MET & PPARG & TP53 \\
AKT1 & CCND1 & ERBB4 & GNAQ & MTOR & PTCH1 & TSC1 \\
ALK & CDK4 & ESR1 & HRAS & MYCN & PTEN & TSC2 \\
AR & CDK6 & FGFR1 & IDH1 & NOTCH1 & RAF1 & \\
BAP1 & CDKN2A & FGFR2 & IDH2 & NRAS & RET & \\
BRAF & DDR2 & FGFR3 & KIT & PDGFRA & ROS1 & \\
BRCA1 & EGFR & FGFR4 & KRAS & PD-L1 & SMO & \\
BRCA2 & ERBB2 & FLT3 & MAP2K1 & PIK3CA & STK11 & \\
\hline
\end{tabular}

Table II. Clinical characteristics of the 20 gynecological cancer patients in this study.

\begin{tabular}{llcc}
\hline Characteristics & Number & Range (\%) \\
\hline Age & Median (IQR) & 62 & $42-91$ \\
Stage & I & 8 & $(40 \%)$ \\
& II & 4 & $(20 \%)$ \\
& III & 5 & $(25 \%)$ \\
Cancer type & IV & 3 & $(15 \%)$ \\
& Ovarian cancer & 9 & $(45 \%)$ \\
& Uterine sarcoma & 3 & $(15 \%)$ \\
& Cervical cancer & 4 & $(20 \%)$ \\
& Endometrial cancer & 3 & $(15 \%)$ \\
& Vulvar cancer & 1 & $(5 \%)$ \\
\hline
\end{tabular}

isolated using CD-PRIMETM system (Clinomics Inc., Suwon, Republic of Korea). DNA extraction of CTCs were followed after a filtration process. One $\mu \mathrm{l}$ of purified DNA from each sample was employed to analyze its quality and size using a Bioanalyzer system (Agilent, Santa Clara, CA, USA). In addition, DNA concentration was assessed using the dsDNA BR assay on Qubit fluorometer (Thermo-Fisher Scientific, Waltham, MA, USA).

Patient and blood samples. A total of 20 patients were diagnosed with gynecologic cancers at Chonbuk National University Hospital. All subjects submitted their informed consent for inclusion before participating in the study. Peripheral blood was collected in 10-ml EDTA vacutainers (BD, Cowley, Oxon, UK) about a week before treatment. The study was conducted in accordance with the Declaration of Helsinki, and the protocol was approved by the Ethics Committee of Chonbuk National University Hospital (IRB No. CUH2017-04-018-001).

Sample preparation. Plasma was isolated from $7 \mathrm{ml}$ of whole blood using density gradient centrifugation in Ficoll-Paque ${ }^{\mathrm{TM}}$ PLUS (GE Healthcare, Piscataway, NJ, USA). cfDNA was extracted from about $4 \mathrm{ml}$ of plasma samples using the QIAamp Circulating Nucleic Acid Kit (Qiagen, Hilden, Germany) following the manufacturer's instructions carefully.

Peripheral blood mononuclear cell (PBMC) layer was processed to enrich CTCs using the CD-PRIME ${ }^{\mathrm{TM}}$ system (Clinomics Inc., Suwon, Republic of Korea). CD-PRIME ${ }^{\mathrm{TM}}$ is a centrifugal-force- 

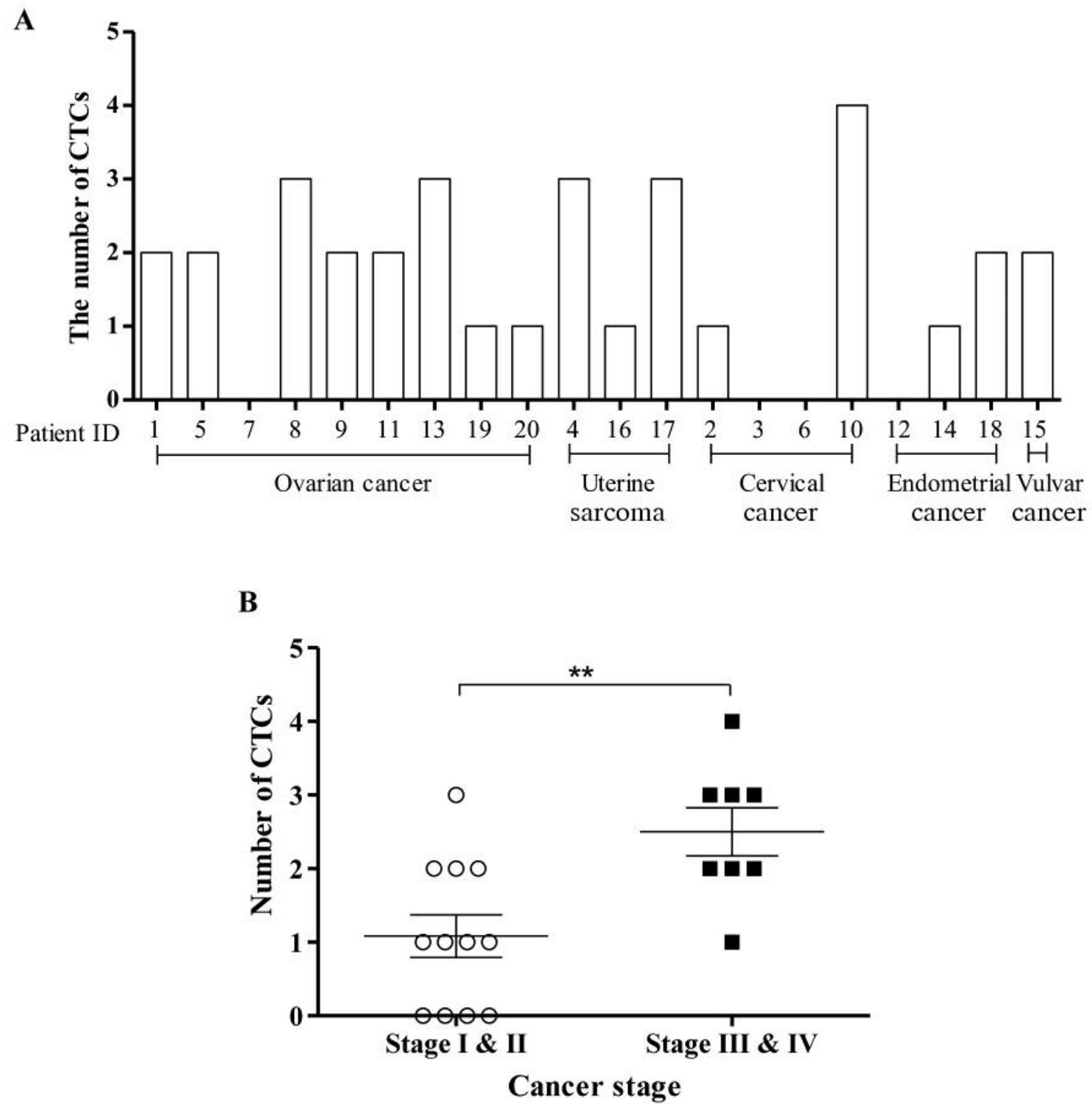

Figure 1. The number of circulating tumor cells (CTCs) in gynecological cancer patients. (A) CTCs isolated from whole blood from the 20 gynecological cancer patients using a size-selective CTC isolation platform. The number of CTCs were quantified by counting anti-EpCAM and anti-cytokeratin among all mononuclear cells. (B) CTC numbers are determined in gynecological cancer patients of different clinical stages (means $\pm S D, * * p<0.005$, Student's $t$-test).

based size-selective CTC isolation platform that can isolate and enrich CTCs from PBMC layer. Following the manufacturer's instructions, an equal volume of Ficoll-Paque ${ }^{\mathrm{TM}}$ PLUS solution was added into the peripheral blood and the corresponding mixture was centrifuged at $800 \times g$. After centrifugation, the PBMC fraction was recovered from the separated blood cell fraction and was applied to the CD-PRIME ${ }^{\mathrm{TM}}$ platform for CTC enrichment (18-20). Based on the general criteria for CTCs identification employing DAPI+, EpCAM/CK+, and CD45-, we were able to calculate the exact number of CTCs using a fluorescence microscope (Bioview, Nes Ziona, Israel) at a magnification of $\times 40$.
By utilizing CD-PRIME ${ }^{\mathrm{TM}}$, enriched CTCs were located on the membrane of CD-CTC-discs provided by the manufacturer. This membrane was placed into collection tubes to be processed with the DNA extraction protocol using QIAamp DNA Micro Kit (Qiagen, Hilden, Germany). The extracted ctcDNA, was further processed by whole genome amplification (WGA) using REPLI-g Mini Kit (Qiagen, Hilden, Germany). We analyzed the quality and estimated the quantity of cfDNA and ctcDNA using the Qubit 3.0 fluorometer (Thermo-Fisher Scientific, Waltham, MA, USA). In addition, the 2100 Bioanalyzer (Agilent, Santa Clara, CA, USA) was employed to detect the degree of genomic DNA contamination. 
A total of $10 \mathrm{ng}$ of cfDNA or ctcDNA were harnessed for the library construction. Library preparation was performed using Ion Ampliseq Library Kit 2.0 (Thermo-Fisher Scientific, Waltham, MA, USA) according to the manufacturer's instruction. We used the Ion Xpress Barcode Adaptors Kit (Thermo-Fisher Scientific, Waltham, MA, USA) for sample multiplexing and the resulting libraries were purified with the Agencourt AMPure XP reagent (Beckman Coulter, Danvers, MA, USA). Synthesized libraries were then quantified using the Qubit 3.0 fluorometer and the 2100 Bioanalyzer. Template preparation for the libraries was performed using the Ion Chef Instrument (Thermo-Fisher Scientific, Waltham, MA, USA) with Ion 540 Chef Kit (Thermo-Fisher Scientific, Waltham, MA, USA). Multiplexed templates were subjected to sequencing on the Ion S5 XL system (Thermo-Fisher Scientific, Waltham, MA, USA).

Panel development. We designed a customized NGS panel to characterize single nucleotide variants (SNVs), insertion-deletion polymorphisms (INDELs), and copy-number variations (CNVs) in 51 actionable genes (Table I). Candidate genes are included on the basis of associated FDA-approved therapies or reported clinical trials. The cancer panel was designed using Ampliseq Designer (5.4.1, Thermo-Fisher Scientific, Waltham, MA, USA). A total of 1,355 amplicons are designed in two primer pools. Amplicon size was determined to lie within the $125-175 \mathrm{bp}$ range and the total number of bases covered by the amplicons was $136.75 \mathrm{~kb}$. The panel performance was assessed by Multiplex 1 cfDNA Reference Standard set (Horizon, Cambridge, UK).

Sequencing data analysis. The hg19 human genome reference was used as the reference. Sequence data analysis was performed using Torrent Suite software (5.8.0). IonTorrent provided plugins such as coverage Analysis (Version 5.8.0.1), variant caller (Ver 5.8.0.19), and Ion Reporter (Ver. 5.10.2.0) were utilized to analyze sequencing coverage, generate VCF files, and obtain annotation of genetic variants respectively. To filter out potential sequencing background noise, we excluded Common Korean SNVs which are included in the KoVariome whole genome sequence database from 50 healthy unrelated Korean individuals $(17,21)$ and any genetic variants with allele frequency below $1 \%$ were treated as random errors and discarded.

Statistical analyses. All data were expressed as mean $\pm \mathrm{SD}$, except indicated \pm SEM in the legends of at least three independent experiments. Two-tailed $t$-tests and linear regression with Pearson correlation coefficient were performed using $\mathrm{GraphPad}^{\mathrm{TM}} 4.0$ software. $p$-values less than 0.05 were used to determine the statistical significance.

\section{Results}

Clinical characteristics of examined patients. We have collected 10-ml blood samples from 20 patients who were diagnosed with gynecological cancer. The baseline characteristics of these patients are demonstrated in Table II. The median age was 62 years old with a range between 42 and 91. Because gynecologic cancer occurs in different parts of female's reproductive organs, patients were classified into five distinct types of gynecological cancer. The most

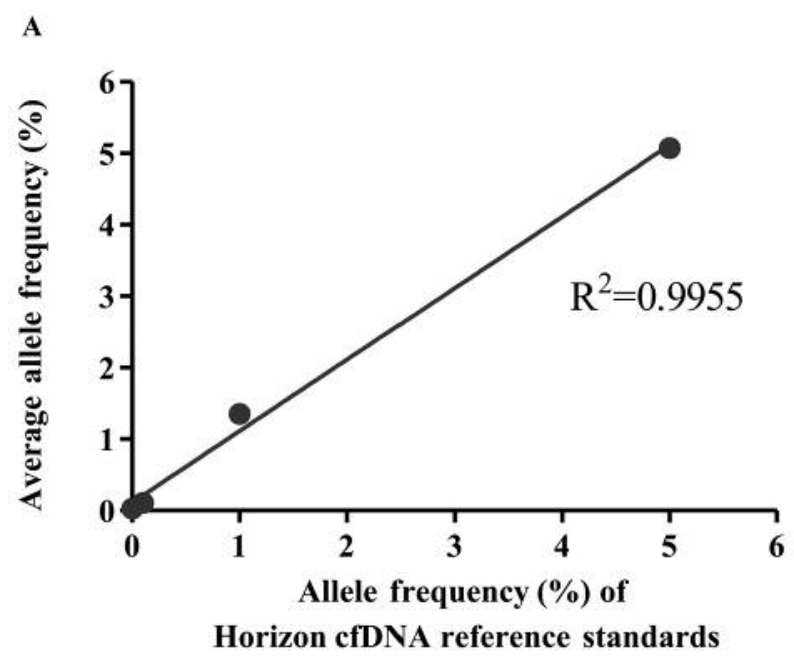

B

\section{EGFR exon19 deletion}

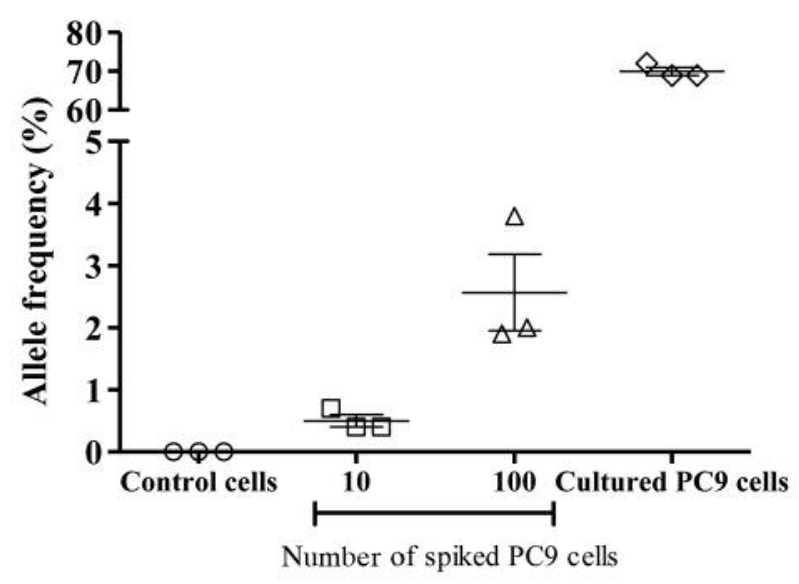

Figure 2. The performance of the customized NGS panel was measured using cfDNA reference standard or the blood spiking PC9 cells, respectively. (A) The average allele frequency of eight variants (triplicate, $y$-axis) as a function of the expected allele frequency (x-axis) for the Horizon cfDNA reference standards. (B) The 10- and 100-PC9 cell pools were generated by serial dilutions. Cultured KOREF cells and PC9 cells used as EGFR exon 19 deleted negative cells and positive cells, respectively.

frequent type was identified as ovarian cancer including high-grade serous carcinoma, low-grade serous carcinoma, endometrioid adenocarcinoma, clear cell carcinoma and adult granulosa cell tumor $(n=9,45 \%)$, followed by uterine sarcoma including leiomyosarcoma, spindle cell sarcoma and carcinosarcoma $(n=3,15 \%)$, cervical cancer including squamous cell carcinoma and adenocarcinoma, $(n=4,20 \%)$, endometrial cancer $(n=3,15 \%)$, and finally vulvar cancer 
A

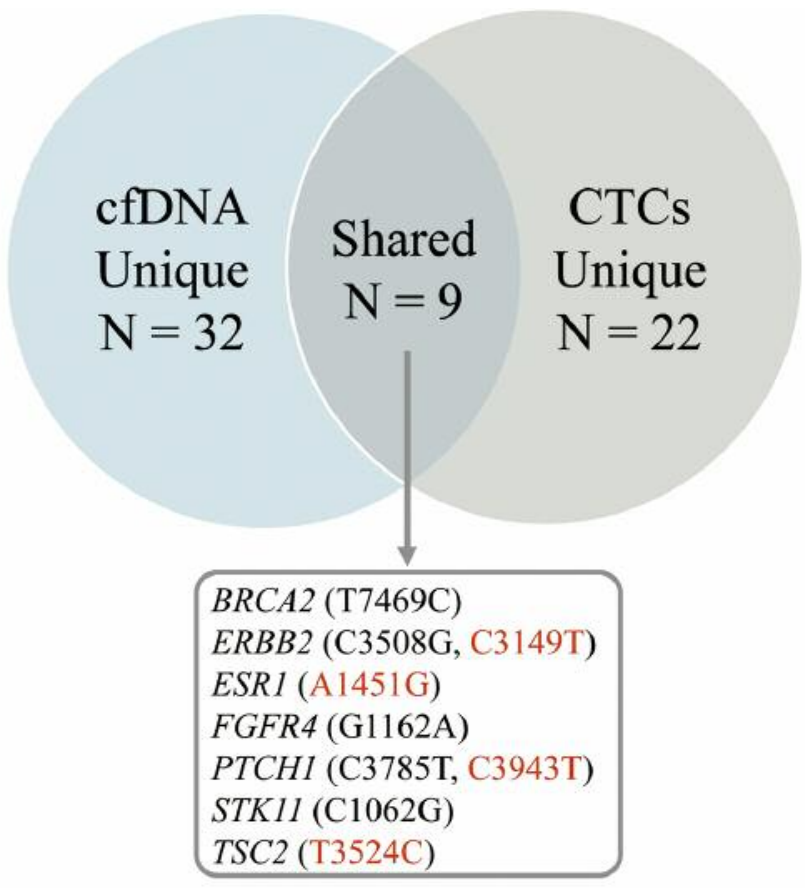

B

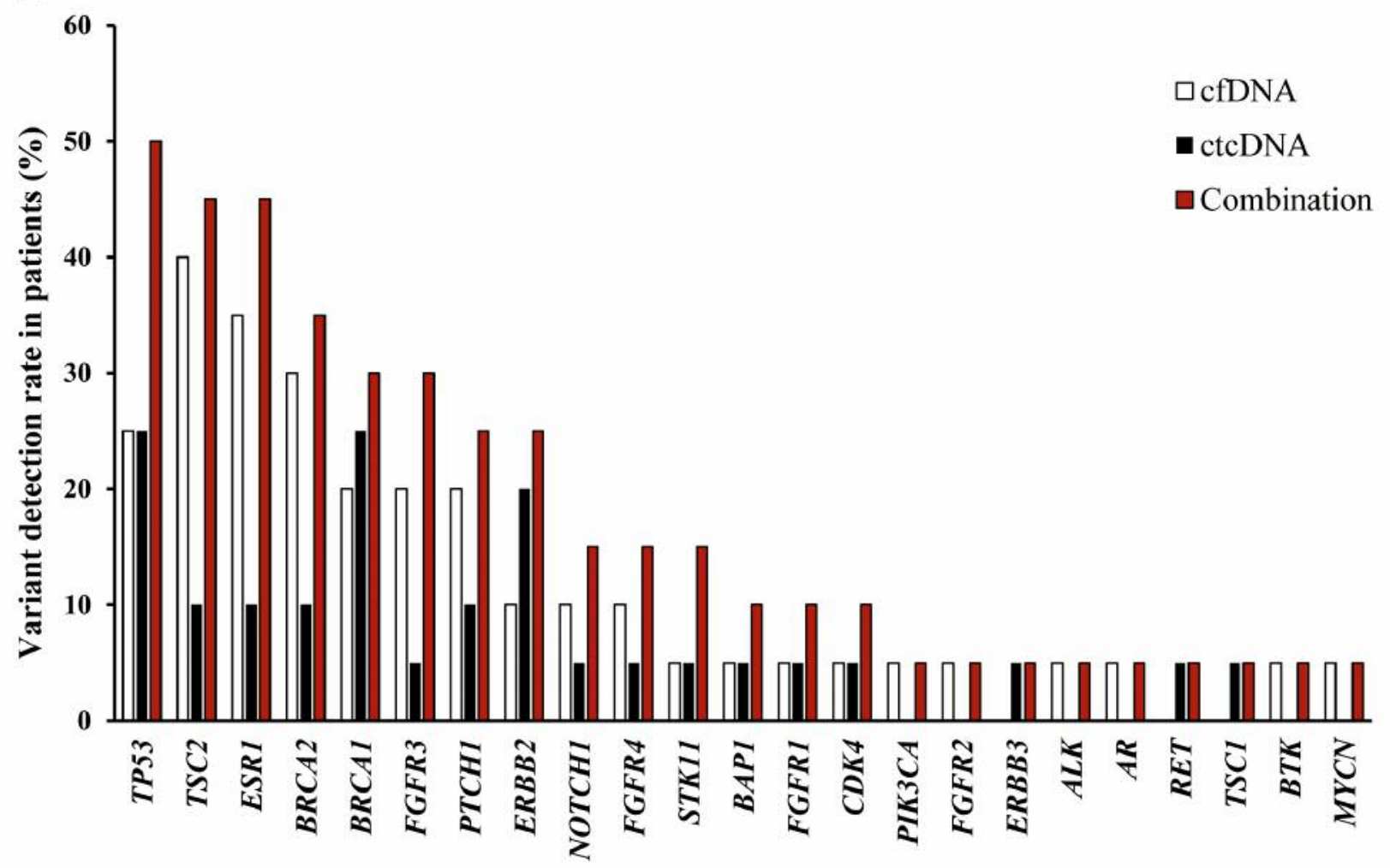

Figure 3. The combinatory analysis of genetic alterations using cfDNA and ctcDNA. (A) Venn diagram of matched cfDNA variants (blue) and ctcDNA (grey). The genetic alterations in cfDNA and ctcDNA in gynecological cancer patients. Nine genetic variants (red) were shared between cfDNA and ctcDNA. Four variants matched in cfDNA and ctcDNA of same patients. (B) The percentage of patients with mutations detected using combination, cfDNA or ctcDNA alone. Multiple genetic variants within the same gene in each patient were counted as one. 
$(n=1,5 \%)$. The number of patients in the four cancer stages was eight at Stage I (40\%), four at Stage II (20\%), five at Stage III (25\%), and three at Stage IV (15\%).

Besides, we filtered out the CTCs from the peripheral blood and counted the number of cells by immunostaining. As a result, 16 out of 20 samples were detected to have a certain number of CTCs and the median number of CTC was 1.65 ranging from 0 to 4 (Figure 1A). The counted CTCs were classified according to the care stage of the patients. As a result, significantly higher numbers were observed in the cancer stage III \& IV group than the stage I \& II group (Figure 1B).

Detection limit of NGS panel using cfDNA and CTCs. To establish the detection limit of the customized NGS panel, we first used the Multiplex I cfDNA Reference Standard set (Horizon Discovery). This reference standard set consists of eight genetic variants at four different expected allele frequencies $(5 \%, 1 \%, 0.1 \%$, and $0 \%)$. Triplicates of each reference material were analyzed with the custom NGS panel. We discovered that the developed NGS panel was able to detect NRAS (Q61K, A59T), EGFR (E756-A750, T790M, L858R), and KRAS (G12D) mutations of up to $0.1 \%$ allele frequencies, and the EGFR (V769 - D770insASV) and PIK3CA (E545K) were able to detect genetic variants of up to $1 \%$ (Figure 2A). Although these results do not completely follow the results demonstrated by the Horizon discovery, they also reported that most of the genetic variants at $0.1 \%$ allelic frequency were detectable only with Droplet Digital PCR, not with NGS. Therefore, it is much more conservative to say that the custom NGS panel has the detection limit of $1 \%$ allelic frequency, even though some variants showed expression at $0.1 \%$ allele frequency.

To further confirm the applicability of detecting genetic mutations in ctcDNA, we employed EGFR exon 19 deleted PC9 cells and prepared two different samples by spiking ten or a hundred cells into the blood of healthy individuals. As a result, mean values of allele frequency of EGFR exon 19 deletion were 0 in KOREF (negative control), $0.50( \pm 0.17)$ in 10spiked PC9 cells, $2.57( \pm 1.07)$ in 100-spiked PC9 cells, and $69.93( \pm 1.79)$ in cultured PC9 cells (positive control, Figure 2B). These allele frequencies were also confirmed by the qualitative qPCR results on mutation detection (data not shown).

Genomic profile difference between cfDNA and CTCs. Using cfDNA and ctcDNA extracted from 20 clinical samples, we performed ultra-deep sequencing with customized NGS panel. Then, we identified 63 genetic variants expressed in 23 actionable genes by comparing the genomic profiles between them. As a result, we observed several unique variants as well as nine variants that were expressed in both cfDNA and ctcDNA (Figure 3A). Among these genes, concurrently expressed variants were ERBB2 S1050L in patient 11, ESR1 D484G and TSC2 V1175V in patient 5, and PTCH1 P1315S in patient 4.

To investigate the clinical application of comprehensive profiling of cfDNA and ctcDNA, we calculated variant detection rates for 23 genes in patients (Figure 3B). TP53 mutations were detected in 10 patients with a simultaneous investigation of cfDNA and ctcDNA genomic profiles. However, the analysis of the individual profiles can only detect five patients and loses the other five patients who were classified as false negatives. Other variants also follow a similar trend, for example, the combined detection rates for TSC2, ESR1, BRCA2, BRCA1, and PTCH1 are 45\%, 45\%, $35 \%, 30 \%$, and $30 \%$, respectively. Also, these detection rates are conspicuously higher than the individual detection rates, improving the overall detection rate of genetic profiles using liquid biopsy.

\section{Discussion}

Despite the fact that many liquid biopsy studies on cancer diagnosis, monitoring, and prognosis have been mainly focused on cfDNA, detecting genetic variants in a minuscule amount of cfDNA is restricted by both the blood volume drawn and the overall detection sensitivity. Obtaining more than $40 \mathrm{ml}$ of blood would improve the detection of mutations in cfDNA, but this is an unfeasible solution in most clinical settings. In addition, the average concentration of cfDNA in healthy individuals is reported to be $5 \mathrm{ng} / \mathrm{ml}$, ranging from 1 to $10 \mathrm{ng} / \mathrm{ml}$. By assuming the mass of one genome copy to be $6.6 \mathrm{pg}$, there are only 6,000 genome copies at max in $4 \mathrm{ml}$ of plasma separated from $10 \mathrm{ml}$ of blood. In 6,000 genome copies, the estimated number of ctDNA is less than a single cancer genome. This indicates that the NGS panel should have sensitivity higher than $0.01 \%$, and that cfDNA detection is limited in the current state of NGS technology.

To overcome the shortcomings posed by cfDNA application, we decided to perform analysis on both cfDNA and ctcDNA. The initial step was to define the lowest number of CTCs that can be detected by the custom NGS panel we proposed. Our result showed that the allele frequency of the EGFR exon 19 deletion at ten cells of spiked PC9 was $0.5 \%$. However, although only 0-4 CTCs were successfully separated from the clinical samples and confirmed by the immune system, genetic variants of ctcDNA were observed. This discrepancy is expected to result from the immunostaining method using EpCAM and cytokeratin antibodies. The EpCAM-, cytokeratin-positive CTC only reflects the subpopulation of the entire population, leading to underestimation of the number of actual CTCs existing in the blood (22). Therefore, the number of isolated CTCs above the filter membrane is expected to be higher than the counting number. Consequently, NGS using isolated CTCs would provide more comprehensive 
information such as heterogeneity of CTCs rather than subpopulation characteristics.

Combinatory analysis of cfDNA and ctcDNA did provide complementary genetic information of tumors that each sample misses. Among 23 genes detected for genetic variants, $B R C A 1$ and $E R B B 2$ were detected in CTC in more patients than in cfDNA, and in ERBB3, RET, and TSC1, gene variations missed by cfDNA were detected in ctcDNA. Especially, TP53 variants were able to cover $25 \%$ of patients when cfDNA or ctcDNA were analyzed separately, while the combinatory analysis of cfDNA and ctcDNA was able to cover half of the cohort. This means that cfDNA used clinically represented only a fraction of the tumor heterogeneity in cancer diagnosis, monitoring and prognosis in patients. Thus, the analysis of CTCs and cfDNA together may provide richer tumor heterogeneity information, which can provide useful information for cancer therapy.

This study is limited in that only 20 patients were enrolled. Therefore, it needs to be validated on a much larger scale of clinical studies. Second, it is difficult to obtain a consistent number of CTCs. In some cases, it was not possible to detect CTCs by immunostaining as four patients showed zero CTC count in our study. Although the detection of CTCs by immunostaining may miss other CTC subpopulations, NGS analysis with high sensitivity can still detect genetic variants. Third, Whole Genome Amplification (WGA) was performed in order to increase the DNA yield, since CTCs contain a minuscule amount of DNA. It is well known that WGA can introduce amplification errors that affect appropriate genetic variant analysis negatively (23). Nevertheless, the kit we have used in our study utilizes a high fidelity Phi29 polymerase which has proofreading activity as well as a very low error rate. Moreover, sequenced data have been rearranged based on the stringent filtering protocol, meaning that variants with allele frequency less than $1 \%$ were treated as errors and eradicated from the variants list. With the approach above, WGA and polymerase errors became negligible.

In conclusion, our NGS based genetic profiling analysis characterized tumors in both cfDNA and ctcDNA from the same patients and we found that such a combined method can offer a better data set for guiding clinical strategies and targeted therapies.

\section{Conflicts of Interest}

The Authors declare no potential conflicts of interest related to this work.

\section{Authors' Contributions}

Conceptualization, SYL, DKC, JA, CHC, SJ, JB, BCK, DHC; data curation, DKC, JA, SCY, SJ, JB; writing-original draft preparation, DKC; writing-review and editing, SYL, SCY, CHC, JB, BCK, DHC; visualization, DKC, SCY, DHC; validation, JB; supervision,
BCK, DHC. All authors finally approved the version to be published and agree to be accountable for all aspects of the work.

\section{Acknowledgements}

This study was partly supported by a grant from the Basic Research Program (2017R1A2B4012353) and the Bio \& Medical Technology Development Program (2017M3A9F7074175) of the Nation Research Foundation (NFR) funded by the Ministry of Science \& ICT. This study was also supported by the Research Base Construction Fund Support Program funded by Chonbuk National University in 2019. This study was part of the Investment-linked Corporate Growth R\&D Support Program (KOITAR\&D150405) by Korea Industrial Technology Association. JB was supported by the Genome Korea Project in Ulsan Research Fund (1.180024.01 and 1.180017.01) of UNIST and the Genome Korea Project in Ulsan.

\section{References}

1 Ready N, Hellmann MD, Awad MM, Otterson GA, Gutierrez M, Gainor JF, Borghaei H, Jolivet J, Horn L, Mates M, Brahmer J, Rabinowitz I, Reddy PS, Chesney J, Orcutt J, Spigel DR, Reck M, O’Byrne KJ, Paz-Ares L, Hu W, Zerba K, Li X, Lestini B, Geese WJ, Szustakowski JD, Green G, Chang H and Ramalingam SS: First-line nivolumab plus ipilimumab in advanced non-small-cell lung cancer (checkmate 568): Outcomes by programmed death ligand 1 and tumor mutational burden as biomarkers. J Clin Oncol 37(12): 992-1000, 2019. PMID: 30785829. DOI: $10.1200 /$ JCO.18.01042

2 Goldberg KB, Blumenthal GM and Pazdur R: The first year of the food and drug administration oncology center of excellence: Landmark approvals in a dynamic regulatory environment. Cancer J 24(3): 131-135, 2018. PMID: 29794538. DOI: 10.1097/PPO.0000000000000316

3 Chen XQ, Stroun M, Magnenat JL, Nicod LP, Kurt AM, Lyautey J, Lederrey $\mathrm{C}$ and Anker P: Microsatellite alterations in plasma DNA of small cell lung cancer patients. Nat Med 2(9): 1033-1035, 1996. PMID: 8782463. DOI: 10.1038/nm0996-1033

4 Buim ME, Fanelli MF, Souza VS, Romero J, Abdallah EA, Mello CA, Alves V, Ocea LM, Mingues NB, Barbosa PN, Tyng CJ, Chojniak R and Chinen LT: Detection of kras mutations in circulating tumor cells from patients with metastatic colorectal cancer. Cancer Biol Ther 16(9): 1289-1295, 2015. PMID: 26252055. DOI: $10.1080 / 15384047.2015 .1070991$

5 Isobe K, Hata Y, Kobayashi K, Hirota N, Sato K, Sano G, Sugino K, Sakamoto S, Takai Y, Shibuya K, Takagi K and Homma S: Clinical significance of circulating tumor cells and free DNA in non-small cell lung cancer. Anticancer Res 32(8): 3339-3344, 2012. PMID: 22843912

6 Heitzer E, Perakis S, Geigl JB and Speicher MR: The potential of liquid biopsies for the early detection of cancer. NPJ Precis Oncol 1(1): 36, 2017. PMID: 29872715. DOI: 10.1038/s41698-0170039-5

7 Thierry AR, Mouliere F, El Messaoudi S, Mollevi C, LopezCrapez E, Rolet F, Gillet B, Gongora C, Dechelotte P, Robert B, Del Rio M, Lamy PJ, Bibeau F, Nouaille M, Loriot V, Jarrousse AS, Molina F, Mathonnet M, Pezet D and Ychou M: Clinical validation of the detection of kras and braf mutations from circulating tumor DNA. Nat Med 20(4): 430-435, 2014. PMID: 24658074. DOI: $10.1038 / \mathrm{nm} .3511$ 
8 Diehl F, Schmidt K, Choti MA, Romans K, Goodman S, Li M, Thornton K, Agrawal N, Sokoll L, Szabo SA, Kinzler KW, Vogelstein B and Diaz LA, Jr.: Circulating mutant DNA to assess tumor dynamics. Nat Med 14(9): 985-990, 2008. PMID: 18670422. DOI: $10.1038 / \mathrm{nm} .1789$

9 Bettegowda C, Sausen M, Leary RJ, Kinde I, Wang Y, Agrawal N, Bartlett BR, Wang H, Luber B, Alani RM, Antonarakis ES, Azad NS, Bardelli A, Brem H, Cameron JL, Lee CC, Fecher LA, Gallia GL, Gibbs P, Le D, Giuntoli RL, Goggins M, Hogarty MD, Holdhoff M, Hong SM, Jiao Y, Juhl HH, Kim JJ, Siravegna G, Laheru DA, Lauricella C, Lim M, Lipson EJ, Marie SK, Netto GJ, Oliner KS, Olivi A, Olsson L, Riggins GJ, Sartore-Bianchi A, Schmidt K, Shih 1 M, Oba-Shinjo SM, Siena S, Theodorescu D, Tie J, Harkins TT, Veronese S, Wang TL, Weingart JD, Wolfgang CL, Wood LD, Xing D, Hruban RH, Wu J, Allen PJ, Schmidt CM, Choti MA, Velculescu VE, Kinzler KW, Vogelstein B, Papadopoulos N and Diaz LA, Jr.: Detection of circulating tumor DNA in early- and late-stage human malignancies. Sci Transl Med 6(224): 224ra224, 2014. PMID: 24553385. DOI: 10.1126/scitranslmed.3007094

10 Schwarzenbach H, Hoon DS and Pantel K: Cell-free nucleic acids as biomarkers in cancer patients. Nat Rev Cancer 11(6): 426-437, 2011. PMID: 21562580. DOI: $10.1038 / \mathrm{nrc} 3066$

11 Fiala C and Diamandis EP: Utility of circulating tumor DNA in cancer diagnostics with emphasis on early detection. BMC Med 16(1): 166, 2018. PMID: 30285732. DOI: 10.1186/s12916-018$1157-9$

12 Wan JCM, Massie C, Garcia-Corbacho J, Mouliere F, Brenton JD, Caldas C, Pacey S, Baird R and Rosenfeld N: Liquid biopsies come of age: Towards implementation of circulating tumour DNA. Nat Rev Cancer 17(4): 223-238, 2017. PMID: 31579300. DOI: $10.20517 / j \operatorname{tgg} .2019 .07$

13 Mouliere F, Robert B, Arnau Peyrotte E, Del Rio M, Ychou M, Molina F, Gongora $\mathrm{C}$ and Thierry AR: High fragmentation characterizes tumour-derived circulating DNA. PLoS One 6(9): e23418, 2011. PMID: 21909401. DOI: 10.1371/journal. pone. 0023418

14 Heitzer E, Auer M, Gasch C, Pichler M, Ulz P, Hoffmann EM, Lax S, Waldispuehl-Geigl J, Mauermann O, Lackner C, Hofler G, Eisner F, Sill H, Samonigg H, Pantel K, Riethdorf S, Bauernhofer T, Geigl JB and Speicher MR: Complex tumor genomes inferred from single circulating tumor cells by array-cgh and next-generation sequencing. Cancer Res 73(10): 2965-2975, 2013. PMID: 23471846. DOI: 10.1158/0008-5472.CAN-12-4140

15 Horton CE, Kamal M, Leslie M, Zhang R, Tanaka T and Razaq M: Circulating tumor cells accurately predicting progressive disease after treatment in a patient with non-small cell lung cancer showing response on scans. Anticancer Res 38(2): 1073-1076, 2018. PMID: 29374743. DOI: 10.21873/anticanres.12325
16 Onidani K, Shoji H, Kakizaki T, Yoshimoto S, Okaya S, Miura N, Sekikawa S, Furuta K, Lim CT, Shibahara T, Boku N, Kato K and Honda K: Monitoring of cancer patients via next-generation sequencing of patient-derived circulating tumor cells and tumor DNA. Cancer Sci 110(8): 2590-2599, 2019. PMID: 31169336. DOI: $10.1111 /$ cas.14092

17 Cho YS, Kim H, Kim HM, Jho S, Jun J, Lee YJ, Chae KS, Kim CG, Kim S, Eriksson A, Edwards JS, Lee S, Kim BC, Manica A, Oh TK, Church GM and Bhak J: An ethnically relevant consensus korean reference genome is a step towards personal reference genomes. Nat Commun 7: 13637, 2016. PMID: 27882922. DOI: 10.1038/ncomms13637

18 Lee A, Park J, Lim M, Sunkara V, Kim SY, Kim GH, Kim MH and Cho YK: All-in-one centrifugal microfluidic device for size-selective circulating tumor cell isolation with high purity. Anal Chem 86(22): 11349-11356, 2014. PMID: 25317565. DOI: 10.1021/ ac5035049

19 Kim TH, Lim M, Park J, Oh JM, Kim H, Jeong H, Lee SJ, Park HC, Jung S, Kim BC, Lee K, Kim MH, Park DY, Kim GH and Cho YK: Fast: Size-selective, clog-free isolation of rare cancer cells from whole blood at a liquid-liquid interface. Anal Chem 89(2): 1155-1162, 2017. PMID: 27958721. DOI: 10.1021/ acs.analchem.6b03534

20 Kang HM, Kim GH, Jeon HK, Kim DH, Jeon TY, Park DY, Jeong H, Chun WJ, Kim MH, Park J, Lim M, Kim TH and Cho YK: Circulating tumor cells detected by lab-on-a-disc: Role in early diagnosis of gastric cancer. PLoS One 12(6): e0180251, 2017. PMID: 28662130. DOI: 10.1371/journal.pone.0180251

21 Kim J, Weber JA, Jho S, Jang J, Jun J, Cho YS, Kim HM, Kim H, Kim Y, Chung O, Kim CG, Lee H, Kim BC, Han K, Koh I, Chae KS, Lee S, Edwards JS and Bhak J: Kovariome: Korean national standard reference variome database of whole genomes with comprehensive snv, indel, cnv, and sv analyses. Sci Rep 8(1): 5677, 2018. PMID: 29618732. DOI: 10.1038/s41598-018-23837-x

22 Kato K, Shoji H, Kakizaki T, Honda K, Kakimoto A, Sakuma T, Yamada T, Fang S, Wu A, Lim CT and Furuta K: Next generation sequencing of circulating tumor cells isolated from the peripheral blood of patients with gastrointestinal cancer. Ann Oncol 25(suppl_4): iv558-iv558, 2014. DOI: 10.1093/annonc/ mdu358.44

23 Macaulay IC and Voet T: Single cell genomics: Advances and future perspectives. PLoS Genet 10(1): e1004126, 2014. PMID: 24497842. DOI: 10.1371/journal.pgen.1004126

Received October 14, 2019

Revised October 30, 2019

Accepted October 31, 2019 